richtet. Dabei werden die im ersten Teil angesprochenen Aspekte aufgegriffen und eingehend diskutiert.

Zusammenfassend bleibt festzustellen, daß das besprochene Buch durchaus dazu geeignet ist, den Leser in die Rolle der Europäischen Gemeinschaft in der Weltwirtschaft einzuführen. Obgleich schon im Hinblick auf den behandelten Themenkreis der Tagung rechtliche Fragen naturgemäß nicht im Vordergrund standen, kann sich doch auch der gemeinschaftsrechtlich interessierte Jurist mit der Lektüre dieses Buches in einigen Bereichen brauchbare und nützliche (Hintergrund-)Informationen verschaffen.

Roger Kampf

\title{
Peter Hammans
}

\section{Das politische Denken der neueren Staatslehre in der Bundesrepublik}

Opladen, Westdeutscher Verlag, 1987, VIII/334 S., DM 49,-

Rechtswissenschaft ist die Wissenschaft vom geltenden Recht. Sie hat die vorhandene Rechtsordnung zum Gegenstand. In diesem Kontext beschäftigt sie sich auch mit den Entstehungs- und Anwendungsbedingungen von Rechtsnormen. Weil sie dies tut, richtet sich ihr Augenmerk auf den Staat. Dieser ist die maßgebliche Instanz der Rechtssetzung und -vollziehung. Und weil er dies ist, ist er auch Endpunkt politischer Interessenartikulation aus der Umwelt und zugleich Ausgangspunkt politischer Interessenvermittlung, die sich in der Rechtsordnung äußert. Ist so der Staat Medium der Transformation politischer Belange in objektives Recht, so gehören Staat und Recht notwendig zusammen. Sicherlich ist dies nur ein Teilaspekt staatlichen Wirkens; aber es ist eben dieser Teilaspekt, welcher den Staat zum Gegenstand der rechtswissenschaftlichen Staatslehre macht. Damit überschneidet sich diese Disziplin hinsichtlich ihres Gegenstandes partiell mit demjenigen der Politikwissenschaft. Eine derartige Duplizität wissenschaftlicher Befassung mit einem einzelnen Gegenstand ist nichts Ungewöhnliches; im Gegenteil. So kann beispielsweise ein einziges Mineral Gegenstand der Geologie, der Chemie, der Physik und der Paläontologie sein, ohne daß solche Vielfalt als problematisch empfunden würde. Das gilt in ähnlicher Weise für den Staat, der nahezu in allen sozial- und den meisten geisteswissenschaftlichen Disziplinen "vorkommt". Günstigstenfalls kann unter solchen Bedingungen die eine von der anderen Disziplin profitieren. Schon unter diesem Aspekt ist eine politikwissenschaftliche Analyse der Staatslehre zu begrüßen.

Das Ergebnis der vorliegenden Untersuchung läßt sich in einem einzigen Satz zusammenfassen: Die juristische Staatslehre verharrt in einem politischen Konservatismus. Wie kommt eine solche Diagnose, die immerhin u.a. das Schaffen von E. W. Böckenförde, F. Ermacora, E. Fraenkel, E. Forsthoff, R. Herzog, K. Hesse, M. Kriele, H. Krüger, 
H. Quaritsch, U. Scheuner, W. Weber und R. Zippelius auf eine einzige, zudem kurze Formel bringt, zustande? Ihre Grundlage sind die Konservatismusthesen Helga Grebings (S. 3 f). Jenen Thesen liegt die Perspektive einer notwendigen historischen "Dynamik des Prozesses der Emanzipation bislang Unterprivilegierter zur Selbstbestimmung und Selbstherrschaft" zugrunde, welche nach wissenschaftlichen Gesetzmäßigkeiten vom Feudalismus über den bürgerlichen Liberalismus hin zur "sozialen Demokratie" voranschreitet. Maßgebliches Kriterium dieses historischen Prozesses ist die fortlaufende Vergrößerung des Bereichs individueller Autonomie durch Abbau gesellschaftlich nicht legitimierter oder legitimierbarer Herrschaft. Ein solcher Prozeß, den Grebing beschreibt und Hammans voraussetzt, ist nicht bloß historisch im Sinne seiner Zugehörigkeit zur Vergangenheit, sondern wirkt auch in der Zukunft fort. Nur wer ihn erkennt, argumentiert historisch; wer anders denkt, denkt "ahistorisch" (so nur statt vieler anderer Beispiele S. 101 gegen Böckenförde!). In einem solchen Modell ist konservativ die "jener Entwicklung der Demokratisierung immanente Gegenbewegung". Dies können Bestrebungen sein, welche das Rad der Geschichte zurückdrehen wollen; aber auch solche, die sich der Gegenwart anpassen, um von hier aus den weiteren Fortschritt zu behindern. Als Vorteile eines solchen, weiten Konservatismusbegriffs werden angegeben: Einerseits seine Weite, der alle möglichen konservativen Bewegungen erfassen kann; andererseits seine Differenziertheit, welche verschiedene Grade von Konservatismus erkennen lasse. Von diesem methodischen Ausgangspunkt des Autors bis zum Ergebnis ist es nicht mehr weit. Da die Juristen den Staat in den Mittelpunkt ihrer "Staatslehre" stellen, denken sie "etatistisch" (S. 24 - 35). Dabei unterscheidet der Autor im Anschluß an Hobbes und C. Schmitt einen "autoritären" und im Anschluß an Locke und R. Smend einen "integrativen" Etatismus (S. 35 - 48). Wer den Staat denkt, denkt notwenig Phänomene wie Staatsgewalt, Souveränität, Herrschaft mit (S. 48 - 71). Frei- oder anders ausgedrückt: pluralistisch - kann dann nicht mehr das Gemeinwesen insgesamt, sondern nur noch der nicht - staatliche Bereich sein (S. 71 - 98). Damit steht das Ergebnis fest: Die Juristen nehmen den Staat einfach hin und trachten nicht nach seiner Uberwindung oder Abschaffung; damit bleibt nur ein "reduziertes" Freiheitsverständnis: Freiheit vom Staat ist für die Juristen notwendig Freiheit im Staat und nicht Freiheit ohne Staat. Damit findet für die Staatslehre die Befreiung der Gesellschaft vom Staat eine notwendige, unübersteigbare Grenze; und eben an dieser muß dann die endgültige Befreiung des Individuums scheitern. Kann der Jurist seinen eigenen Voraussetzungen nach den historischen Prozeß der Befreiung nicht zu Ende denken, so ist er an irgend einem Punkt notwendig konservativ. Was zu beweisen war. Obwohl die Conclusio nach den Prämissen des Autors zwingend ist, verifiziert er sie noch am Beispiel von fünf Politikfeldern: der "streitbaren Demokratie" (S. 117 - 141), der Dichotomie von Rechtsstaat und Sozialstaat (S. 141 159), der Partizipationsdiskussion (S. 159 - 174), dem Parteien- oder Verbändestaat (S. 174 - 206) und der wirtschaftspolitischen Interpretation des Grundgesetzes (S. 206 235). Was immer die Juristen beginnen, es geht nicht ohne Staat oder schafft diesen jedenfalls nicht ab; und deshalb ist es konservativ. Die Zuordnung der einzelnen Strömun- 
gen in der Staatslehre zu den verschiedenen Ausprägungen politikwissenschaftlicher Konservatismusmodelle verläuft dann auch reibungslos: Der autoritäre Etatismus wird dem autoritären Status - quo - Konservatismus, der integrative Etatismus dem Reformkonservatismus zugeordnet (S. 267 - 289). Das Fazit lautet dann, daß "die juristische Staatslehre bisher gegen einen Abbau der Herrschaftsqualität des Status quo der bürgerlich - kapitalistischen Konkurrenzgesellschaft optiert" (S. 289).

Wie die Ergebnisse, so liegen auch die Probleme der Untersuchung bereits in ihrem Ansatz. Die geschichtsteleologische Extrapolation vergangener Prozesse in die Zukunft hat sich in der Philosophie nicht als wahrheitsfähig erwiesen. Die Option für oder gegen ein derartiges Modell ist dann auch keine wissenschaftliche; sie kann nur außerwissenschaftlich begründet sein. Letztlich resultiert dann die Stellungnahme zu dieser Frage in dem "richtigen" oder "falschen" Bewußtsein. Daß die Juristen für den Autor auf der "falschen" Seite stehen, bedarf keiner Erwähnung; die Frage kann für ihn nur sein, warum sie dort stehen. Maßgeblich sind dafür drei Gründe (S. 13 - 17): die soziale Herkunft der Juristen, deren Sozialisation die Anerkennung von Autorität, Kompetenz und Hierarchie einschließt; ihre Ausbildung, die mangelnde, "intellektuelle Selbständigkeit" und "individuelle Motivation" hervorbringt; schließlich die unter ihnen "herrschende Ideologie", welche Herrschaft neutralisiert und entpolitisiert und so eine "Staatsideologie" erst züchtet, welche dann einem Staatsabbau entgegensteht. Angesichts derartiger, wahrhaft beklagenswerter Zustände bleibt aber dann noch die Frage offen, warum sich die aufgeklärte Politikwissenschaft denn überhaupt noch mit der unaufgeklärten Rechtswissenschaft abgibt. Hier allerdings stößt man bei Hammans auf erhebliche Unsicherheiten. Schon bei der Exposition seiner eigenen Grundlagen, eben Helga Grebings Konservativismusthese, teilt er deren Kritiker in 2 Gruppen ein: Einerseits Konservative, andererseits orthodoxe Marxisten (S. 4 f.). Erstere sehen sich pauschalem Ideologieverdacht ausgesetzt (S. 20). Wesentlich defensiver verläuft die Auseinandersetzung mit letzteren. Hier bleibt als Ergebnis übrig, daß eine "Wiederaneignung des Marxismus" geboten sei, verbunden mit "konzeptioneller Uberarbeitung" und "kategorialer Neuinterpretation" (S. 6). Offenbar kommt die Verunsicherung von dieser Seite. Das dort prognostizierte Absterben des Staates findet nach wie vor nicht statt. Auch der vielbeschworene "Spätkapitalismus" zeigt zwar allerlei Tendenzen zur Transformation, aber auch er verschwindet nicht. Hier geht es tatsächlich um Uberarbeitung und Neuinterpretation. Soll man "ahistorisch" im marxistischen Sinne das Fortschrittsdogma aufgeben und sich so seine Fragen und Antworten von der ungeliebten Wirklichkeit vorschreiben lassen? Oder soll man die Theorie unangetastet lassen und die "falsche" Realität dadurch hinwegzuinterpretieren suchen, daß man nach fortschrittsfeindlichen Kräften forscht? Letzterer Weg ist derjenige Hammans. Er hat den behindernden Faktor bei der Staatslehre ausgemacht. So geht es ihm im Ergebnis nicht um "Politikwissenschaft contra Staatslehre". Sein Ziel ist vielmehr, eine Theorie zu rechtfertigen, welche durch die Praxis nicht bestätigt wird. So kann - ungeachtet möglicher marxistischer Glaubenszweifel - ein Dogma aufrechterhalten bleiben, welches sich an der Wirklichkeit nicht stört. In diesem Sinne 
kann das vorliegende Buch im Sinne Kurt Tucholskys als Beitrag zu einem dialektischen Materialismus gewertet werden, welcher erklärt, wie es gehen wird; und wenn es nicht so geht, erklärt er, warum es nicht so geht. Für die Wirklichkeit ist die These vom historischen Fortschritt so irrelevant; sie erklärt nichts und beweist nichts, weil sie selbst unbewiesen und vage ist. Und eben so irrelevant ist dann auch die These, "die Staatslehre" sei konservativ. Aber dies ist nur die eine Seite. Umgekehrt gilt auch: Eine Rechtswissenschaft, welche sich lange als Sozialwissenschaft verstanden hat, muß sich selbstverständlich von den jüngeren, neuen Wissenschaften auf ihre fortdauernde Notwendigkeit befragen lassen. Gesichert ist ihre Existenz nur da, wo sie es schon früher war: als Geisteswissenschaft. Und als solche braucht sie sich auch auf den Glaubenskampf der politischen Ideen nicht einzulassen. Als Ergebnis bleibt festzuhalten: Rechtswissenschaft hat die Aufgabe, geltendes Recht zu erklären. Als solche Disziplin kann sie nicht nur irrelevant sein. Staatslehre muß vielmehr relevant sein. Um ihre Aufgabe besser erfüllen zu können, braucht sie eine relevante Politikwissenschaft. Vielleicht stellt sich dann eines Tages heraus, daß einzelne Staatslehren konservativ sind. Dann stellt sich vielleicht auch die Differenziertheit ein, welche die vorliegende Untersuchung fordert, aber nicht einlöst. Deren Beitrag zur neomarxistischen Binnendiskussion soll hier ungewürdigt bleiben. Einen Beitrag zur Erkenntnis von Staat und Recht leistet sie nicht. Fortschrittliche Gesinnung allein ist noch keine wissenschaftliche Erkenntnis. Wer hierfür noch einen Beleg sucht, findet ihn in Hammans Buch.

Christoph Gusy 